\title{
Alterações musculoesqueléticas pós COVID-19: revisão bibliográfica
}

\author{
Post COVID-19 musculoskeletal changes: bibliographic review \\ Cambios musculoesqueléticos posteriores a COVID-19: revisión bibliográfica
}

Recebido: 25/10/2021 | Revisado: 05/11/2021 | Aceito: 06/11/2021 | Publicado: 25/11/2021

Larissa dos Santos Nascimento Oliveira
ORCID: https://orcid.org/0000-0001-5207-961X
Universidade de Gurupi, Brasil
E-mail: larissaa_santos@hotmail.com
Morgane Ribeiro de Aquino Macedo
ORCID: https://orcid.org/0000-0002-5537-1621
Universidade de Gurupi, Brasil
E-mail morgane@unirg.edu.br

\begin{abstract}
Resumo
A disseminação do COVID-19 em 2020 foi a ameaça à saúde mais séria do mundo desde a chamada Gripe Espanhola em 1918. Com quase todos os países afetados no mundo, 100 milhões de casos confirmados, mais de 2 milhões de mortes e uma contração significativa no mundo produto interno bruto, a pandemia trouxe enormes custos humanos e econômicos. Sendo assim, o objetivo deste artigo é relatar as consequências musculoesqueléticas pós COVID-19, através de revisão de literatura. Os programas de reabilitação devem ser adaptados à gravidade da doença, faixa etária, níveis de condicionamento físico anteriores e comorbidades pré-existentes. Para a reabilitação adequada de pacientes, necessitarão de novos conhecimentos e habilidades sobre o COVID-19. Logo, espera-se que pacientes acometidos por COVID- 19 sofram consequências musculoesqueléticas em decorrência do processo inflamatório e perca de massa óssea e muscular ocasionada pela imobilidade, gerando incapacidades motoras ainda não quantificáveis. É importante entender as implicações clínicas causadas pelo COVID-19, a fim de se ter melhores estratégias de reabilitação para esses pacientes. O objetivo deste estudo foi realizar uma análise reflexiva do impacto do COVID-19 no sistema imunológico, neuromuscular e musculoesquelético e seu processo de reabilitação.
\end{abstract}

Palavras-chave: Fisioterapia; COVID-19; Reabilitação.

\begin{abstract}
The spread of COVID-19 in 2020 was the most serious health threat in the world since the so-called Spanish Flu in 1918. With almost every country affected in the world, 100 million confirmed cases, more than 2 million deaths and a significant contraction in the world's gross domestic product, the pandemic brought enormous human and economic costs. Thus, the aim of this article is to report the musculoskeletal consequences after COVID-19, through a literature review. Rehabilitation programs must be adapted to the severity of the disease, age group, previous fitness levels and pre-existing comorbidities. For the proper rehabilitation of patients, they will need new knowledge and skills about COVID-19. Therefore, it is expected that patients affected by COVID- 19 suffer musculoskeletal consequences as a result of the inflammatory process and loss of bone and muscle mass caused by immobility, generating motor disabilities that have not yet been quantified. It is important to understand the clinical implications caused by COVID-19 in order to have better rehabilitation strategies for these patients. The aim of this study was to conduct a reflective analysis of the impact of COVID-19 on the immune, neuromuscular and musculoskeletal system and its rehabilitation process.
\end{abstract}

Keywords: Physiotherapy; COVID-19; Rehabilitation.

\section{Resumen}

La propagación del COVID-19 en 2020 fue la amenaza para la salud más grave en el mundo desde la llamada Gripe española en 1918. Con casi todos los países del mundo afectados, 100 millones de casos confirmados, más de 2 millones de muertes y una contracción significativa en el producto interno bruto mundial, la pandemia trajo enormes costos humanos y económicos. Así, el objetivo de este artículo es reportar las consecuencias musculoesqueléticas posteriores al COVID-19, a través de una revisión de la literatura. Los programas de rehabilitación deben adaptarse a la gravedad de la enfermedad, el grupo de edad, el estado físico previo y las comorbilidades preexistentes. Para la rehabilitación adecuada de los pacientes, necesitarán nuevos conocimientos y habilidades sobre COVID-19. Por tanto, se espera que los pacientes afectados por COVID-19 sufran consecuencias musculoesqueléticas como consecuencia del proceso inflamatorio y la pérdida de masa ósea y muscular provocada por la inmovilidad, generando discapacidades motoras que aún no han sido cuantificadas. Es importante comprender las implicaciones clínicas causadas por COVID-19 para tener mejores estrategias de rehabilitación para estos pacientes. El objetivo de este estudio fue realizar un análisis reflexivo del impacto del COVID-19 en el sistema inmunológico, neuromuscular y musculoesquelético y su proceso de rehabilitación.

Palabras clave: Fisioterapia; COVID-19; Rehabilitación. 


\section{Introdução}

A disseminação do COVID-19 em 2020 foi a ameaça à saúde mais séria do mundo desde a chamada Gripe Espanhola em 1918. Com quase todos os países afetados no mundo, 100 milhões de casos confirmados, mais de 2 milhões de mortes e uma contração significativa no mundo produto interno bruto (PIB), a pandemia trouxe enormes custos humanos e econômicos. Como todos nós rapidamente compreendemos, a transmissão do vírus ocorre em grande parte em ambientes fechados com distância espacial reduzida entre os indivíduos (OMS, 2020).

O SARS-CoV, denominado Novel Coronavirus-Infected Pneumonia (NCIP), foi confirmado por meio do lavado bronco-alveolar, sequenciamento de genoma inteiro, PCR e cultura nos pacientes hospitalizados em Wuhan, China, morcegos e outros animais selvagens. A Organização Mundial de Saúde (OMS) confirmou a circulação do novo Coronavírus em 9 de janeiro de 2020, e, desde então, a doença se propagou intensamente pelo mundo (Arora; Grey, 2020).

Portanto, os locais de trabalho, bem como as residências, escolas e outros espaços públicos fechados, logo foram identificados como ambientes nos quais o contágio poderia se espalhar rapidamente. A partilha de transporte ou alojamento também foi identificada como um fator que pode aumentar o risco de contágio (Silva; Dos Santos; De Oliveira, 2020).

Nesse seguimento, as consequências musculoesqueléticas do COVID-19 ainda não foram determinadas, mas sabe-se que os pacientes que necessitam de hospitalização têm maior probabilidade de apresentar atrofia e fraqueza muscular devido à imobilidade e ventilação mecânica prolongada (Fiorillo; Gorwood, 2020).

O SARS-CoV-2 também pode causar disfunções gastrointestinais, hepáticas e renais, além de consequências hematológicas como trombose venosa profunda e tromboembolismo pulmonar, alterações reumatológicas, endócrinas, dermatológicas, psicológicas e cognitivas. No sistema musculoesquelético, condições como sono insuficiente, imobilidade, medo, ansiedade e depressão resultantes de complicações da SARS-CoV-2 e permanência prolongada na UTI podem ser potenciais para o início ou exacerbação da dor pós viral persistente (Bentes et al., 2021).

O objetivo deste artigo é relatar as consequências musculoesqueléticas pós COVID-19, através de revisão de literatura.

\section{Metodologia}

Trata-se de uma pesquisa de revisão da literatura, de metodologia descritiva, apresentada de modo qualitativo. Segundo Soares et al., (2014) configura-se como um tipo de revisão da literatura que reúne achados de estudos desenvolvidos mediante diferentes metodologias, permitindo aos revisores sintetizar resultados sem ferir a filiação epistemológica dos estudos empíricos incluídos.

Portanto, um estudo integrativo configura-se como uma espécie de revisão de literatura, que pode coletar resultados de pesquisas desenvolvidas por diferentes métodos, possibilitando assim os revisores sintetizar os resultados, sem comprometer uma conexão epistemológica da pesquisa empírica contida. Para conduzir esse processo de maneira lógica, a revisão integrativa exige que os revisores, analisem e sintetizem os dados originais de maneira sistemática e rigorosa (Souza; Silva; Carvalho, 2016).

O método qualitativo é considerado o trabalho mais adequado para a visão de pessoas e grupos, estando perfeitamente adaptado às características destinadas a responder a questões de personalidade e particularidade, pois é caracterizado pelas ciências sociais que não pode ser quantificado. Portanto, esse método atua sobre o universo de causas, crenças, significados, atitudes, ideais e valores que produzem respostas importantes, explicativas e profundas que têm a capacidade de gerar novos relatos não adequados para dados quantitativos (Souza; Silva; Carvalho, 2016).

A coleta de dados foi realizada mediante busca eletrônica, nas bases de dados National Library of Medicine (PUBMED), Scientific Electronic Library Online (SCIELO), Literatura Latino-Americana e do Caribe em Ciências da Saúde (LILACS) e Google Scholar. A temática em questão, foi desenvolvida no período de 2010 a 2021, tendo o enfoque de pesquisa voltado para a questão norteadora que "Quais as consequências musculoesqueléticas que o novo Corona Vírus trouxe para a população?’. 
Para compor a buscas dos dados, foram utilizados os Descritores de Ciências da Saúde (DeCS): Consequências. COVID-19. Intervenção. Os operadores booleanos OR e AND também são usados junto aos descritores.

Para os critérios de inclusão foram utilizados artigos completos em língua portuguesa e inglesa, disponíveis eletronicamente e que obedeçam à temática supracitada. Referente aos critérios de exclusão estão: relatos de experiência, estudos de revisão, livros e pesquisas duplicadas, monografias e dissertações.

Assim, para análise de conteúdo e classificação dos artigos foram seguidos os seguintes passos, segundo Bardin (1997):

a) Pré-análise: leitura flutuante do material coletado; constituição do corpus da pesquisa;

b) Exploração do material: recorte em unidades de registro de contexto; codificação e classificação segundo categorias empíricas e teóricas;

c) Tratamento dos dados e interpretação: análise final dos dados obtidos.

Mediante leitura e análise dos estudos, foi possível se estabelecer um conjunto de pesquisas sobre a temática.

\section{Resultados e Discussão}

Longas hospitalizações, isolamentos e até mesmo distâncias sociais afetam a homeostase muscular, com impacto secundário da inatividade física e do desuso. A causa da perda de massa muscular, provavelmente, é multifatorial, envolvendo inflamação, imobilização, nutrição insuficiente e administração de corticosteroides (Mainardi et al., 2021).

$\mathrm{Na}$ fase crítica da doença e com longa permanência em unidades de terapia intensiva, ocorre perda da homeostase entre a síntese e degradação proteica com redução gradual da renovação muscular. O aumento da degradação da proteína muscular se deve à ação das vias de sinalização intracelular. O sistema ubiquitina-proteassoma, principal via relacionada ao mecanismo de proteólise, possui duas enzimas específicas relacionadas ao processo de atrofia muscular esquelética, ativadas em resposta à inatividade e ao processo inflamatório: atrogina-1 (Muscle Atrophy Fbox) e MuRF- 1 (Muscle Ring Finger -1) (Nagamine; Lourenço; Chaves, 2021).

Poulsen et al. (2012) enunciam que, pacientes internados em UTI apresentam perda de $20 \%$ da massa muscular da coxa na primeira semana de internação. A inflamação associada à imobilidade é mais pronunciada nesta fase, onde as alterações metabólicas explicam o maior índice de percas no início.

No decorrer da internação, os músculos, principalmente os dos membros inferiores, não são expostos a descargas mecânicas com atividade neuromuscular reduzida, o que intensifica uma resposta de adaptação, síntese proteica lenta, maior degradação de proteínas, apoptose das células musculares (principais mecanismos de hipotrofia) e diminuição da musculatura força. Em indivíduos saudáveis expostos à imobilização (repouso no leito), ocorre diminuição da massa (14\%) e da força muscular (16\%). Assim, é possível deduzir que um processo inflamatório causado pela sepse associada ao imobilismo, pode promover perda muscular, até 10 vezes maior do que em pessoas saudáveis (Domenis et al., 2021).

O sistema muscular esquelético se adapta à inatividade física prolongada, fazendo com que fibras musculares fiquem menores (atrofia), além da perda da função e qualidades musculares. As proteínas mecanossensoriais, que permitem às fibras musculares detectar forças mecânicas, também estão envolvidas na regulação da massa muscular esquelética. Sua ativação, durante a contração muscular, regula a renovação proteica por meio da interação com a proteína alvo mecanicista da rapamicina (mTORC1) e com as principais vias proteolíticas: o proteassoma e os sistemas de ubiquitina lisossomal / autofágico (Domenis et al., 2021).

Prejuízos funcionais, comumente relatados na literatura em pacientes críticos, estão diretamente relacionados ao tempo de permanência na UTI e à ventilação mecânica prolongada. Sete dias de repouso na cama já podem reduzir a força muscular em $30 \%$, com uma perda adicional de $20 \%$ da força restante a cada semana. As deficiências na função física e na capacidade de exercício podem durar anos após a alta da UTI. O desuso e a perda da inervação em doenças ou lesões afetam diretamente o 
sistema musculoesquelético promovendo um declínio na massa muscular e força articular e atrofia difusa e musculatura esquelética estriada simétrica apendicular e axial (França et al., 2020).

As intervenções de reabilitação respiratória e neuromuscular, que preconizam o menor tempo possível para intubação e a melhora do estado muscular estão diretamente associadas ao prognóstico dos pacientes internados na UTI. As evidências desse tipo de intervenção no COVID-19 ainda são escassas. Pacientes internados em UTI durante epidemias anteriores sofreram lesões musculoesqueléticas e complicações que necessitaram de reabilitação, com intervenção individualizada e dinâmica, adaptandose às rápidas mudanças que caracterizam a progressão da doença, principalmente nos primeiros sete dias de evolução (Borghi; Sanches, 2021).

Embora COVID-19 afete predominantemente o sistema respiratório, as evidências indicam uma doença multissistêmica grave e letal. As sequelas de longo prazo ainda não são conhecidas, mas as evidências de surtos anteriores de coronavírus demonstram comprometimento motor funcional e respiratório, desgaste emocional e perda de qualidade de vida. As complicações musculoesqueléticas com piora das aptidões físicas são referidas: como ossificação heterotópica, perda de massa muscular, dor prolongada, fraqueza e dispneia. Estima-se que $45 \%$ dos pacientes com alta hospitalar necessitarão de cuidados de saúde e assistência social e 4\% necessitarão de programa de reabilitação (De Souza et al., 2020).

\subsection{Reabilitação}

A reabilitação dos pacientes com COVID-19 começa na admissão para manter o funcionamento dos sistemas vitais e continua na fase pós-admissão para resolver as sequelas e complicações causadas pelo vírus e um longo período de hospitalização (Nogueira et al., 2021).

Durante a internação, as mobilizações precoces na unidade de terapia intensiva para prevenir e reduzir a poli neuromiopatia do paciente crítico, melhora a qualidade de vida, diminui o tempo de internação e diminui a mortalidade durante a internação. O início precoce de um programa estruturado de reabilitação contribui para a otimização da função cognitiva, respiratória, neuromuscular e osteoarticular, encurtando o tempo de permanência na UTI e suas sequelas clínicas e funcionais (Huang et al., 2020).

A prevenção de incapacidades em pacientes criticamente enfermos auxilia no manejo clínico do paciente. Os protocolos de intervenção física e cognitiva melhoram a compreensão do paciente sobre o tratamento e os programas de apoio psicossocial, a mudança de comportamento e a adesão às diretrizes (Nogueira et al., 2021).

Os programas de reabilitação devem ser adaptados à gravidade da doença, idade do paciente, níveis de condicionamento físico anteriores e comorbidades pré-existentes. Alguns componentes essenciais para a reabilitação de pacientes com COVID19, necessitarão de novos conhecimentos e habilidades sobre o COVID-19 (Cunha; Silva, 2021).

O programa de reabilitação deve começar com exercícios físicos de baixa intensidade, com monitoramento contínuo da oxigenação e da fadiga. Se o indivíduo tiver sintomas muito leves que podem ou não ser causados por COVID-19, atividades leves são recomendadas. Além disso, é necessário evitar o sedentarismo por longos períodos (De Holanda et al., 2021).

Durante o exercício físico, os períodos de descanso podem ser aumentados se os sintomas piorarem. Em pessoas que apresentaram sintomas leves ou moderados, exercícios de alongamento e treinamento de força de baixa intensidade são recomendados antes das sessões de treinamento aeróbio direcionado. Em pessoas assintomáticas que tiveram contato com pessoas COVID-19 positivas, a atividade deve ser continuada normalmente. O manejo da dor deve ser centrado no paciente, envolvendo reeducação postural. Os programas de reabilitação física ambulatorial variam de acordo com a necessidade de cada paciente, mas podem durar de seis a 12 semanas e precisam estar associados à reabilitação cognitiva (De Holanda et al., 2021). 


\section{Considerações Finais}

Milhões de indivíduos no mundo estão sendo afetadas pelo SARS-CoV 2. Tem sido uma grande e dolorosa experiência de aprendizado sobre como lidar melhor com doenças graves e letais sem drogas e vacinas. Maneiras de diminuir o contágio e a propagação, seus agravamentos dos sintomas, como prevenir sequelas respiratórias, físicas e psicológicas e, finalmente, como reabilitar e devolver a vida normal aos afetados. Compreender as consequências no pós-pandemia e ofertar o tratamento adequado aos afetados é o grande desafio que deve ser enfrentado com conhecimentos e evidências científicas.

Alguns dos efeitos já são conhecidos e necessitam ser tratados de maneira adequada segundo a necessidade de cada paciente, mas sem perder de vista as características da SARS-CoV 2, que podem exigir cuidados e tratamentos diferenciados. É possível elencar que própria doença e o tratamento necessário podem gerar graves incapacidades e que um tratamento em tempo hábil pode ser imprescindível para a adequada reabilitação dos pacientes.

\section{Referências}

Arora, T., \& Grey, I. (2020). Health behaviour changes during COVID-19 and the potential consequences: A mini-review. Journal of Health Psychology, 25(9), 1155-1163.

Bentes, C. G., Araújo, M. D., Caetano, M. E. N., \& de Holanda, V. B. T. (2021). Incidência de pericardite pós COVID-19 em pacientes de uma clínica cardiológica, no período de março a junho de 2020. Revista Eletrônica Acervo Saúde, 13(6), e7350-e7350.

Borghi-Silva, A., Krishna, A. G., \& Garcia-Araujo, A. S. (2021). Importância da avaliação da capacidade funcional e do exercício físico durante e após a hospitalização em pacientes com COVID-19: revisitando a reabilitação pulmonar.

Cunha, G. F., \& Silva, D. E. M. D. (2021). Reabilitação fisioterapêutica pós-COVID-19.

De Holanda, E. P., De Figueirêdo, T. B. J. C., Alves, N. B. F., De Aragão, E. S., \& de Albuquerque Britto, D. B. L. (2021). Alterações neuromusculares em pacientes com COVID-19. Fisioterapia Brasil, 22(3), 469-485.

de Souza, E. C, \& Reabilitação, A. V. D. (2020). Impactos da Covid-19 na Aptidão Cardiorrespiratória: Exercícios Funcionais e Atividade Física. Revista Brasileira de Atividade Física \& Saúde-RBAFS.

Domenis, L. A. M., Daher, D. V., de Almeida, B. S. S., Araújo, M. C., de Macedo Antunes, J., \& Ferrari, M. F. M. (2021). O desafio do cuidado do enfermeiro ao paciente com lesão musculoesquelética que vivencia a COVID-19: relato de experiência. Research, Society and Development, 10(3), e25110312947e25110312947.

Fiorillo, A., \& Gorwood, P. (2020). The consequences of the COVID-19 pandemic on mental health and implications for clinical practice. European Psychiatry, 63(1).

França, E. F., Macedo, M. M., de França, T. R., Cossote, D. F., Gonçalves, L., dos Santos, P. A. C., \& Miyake, G. M. (2020). Triagem de saúde para participação nos programas de exercício físico pós-pandemia de COVID-19: uma ação necessária e emergente ao profissional de educação física. InterAmerican Journal of Medicine and Health, 3 .

Huang, C., Wang, Y., Li, X., Ren, L., Zhao, J., Hu, Y., \& Cao, B. (2020). Clinical features of patients infected with 2019 novel coronavirus in Wuhan, China. The lancet, 395(10223), 497-506.

Mainardi, E. M., Lima, A. C. C., Pereira, C. V., De Viveiros, M. C., da Silva, P. K. E., \& da Serra Braga, S. D. A. (2021). Protocolo de reabilitação cardiorrespiratória no paciente pós-covid: relato de experiência. Brazilian Journal of Health Review, 4(1), 1049-1052.

Nagamine, B. P., Lourenço, L. K., \& Chaves, C. T. D. O. P. (2021). Recursos fisioterapêuticos utilizados no Pós-COVID 19: Uma revisão bibliográfica. Research, Society and Development, 10(7), e42910716785-e42910716785.

Nogueira, T. L., da Silva, S. D. A., da Silva, L. H., Leite, M. V. S., da Rocha, J. F. A., \& Andreza, R. S. (2021). Pós covid-19: as sequelas deixadas pelo SarsCov-2 e o impacto na vida das pessoas acometidas. Archives of Health, 2(3), 457-471.

Poulsen, J. B. (2012). Impaired physical function, loss of muscle mass and assessment of biomechanical properties in critical ill patients. Dan Med J, 59(11), B4544.

Silva, H. G. N., dos Santos, L. E. S., \& de Oliveira, A. K. S. (2020). Efeitos da pandemia do novo Coronavírus na saúde mental de indivíduos e coletividades/Effects of the new Coronavirus pandemic on the mental health of individuals and communities. Journal of Nursing and Health, 10(4). 